\title{
High resolution numerical study of Rayleigh-Taylor turbulence using a thermal lattice Boltzmann scheme
}

\author{
L. Biferale, ${ }^{1}$ F. Mantovani, ${ }^{2}$ M. Sbragaglia, ${ }^{3}$ A. Scagliarini, ${ }^{1}$ F. Toschi, ${ }^{4}$ and R. Tripiccione ${ }^{5}$ \\ ${ }^{1}$ Department of Physics and INFN, University of Tor Vergata and International Collaboration \\ for Turbulence Research, Via della Ricerca Scientifica 1, 00133 Rome, Italy \\ ${ }^{2}$ Deutsches Elektronen-Synchrotron, Platanenallee 6, 15738 Zeuthen, Germany \\ ${ }^{3}$ Department of Physics and INFN, University of Tor Vergata, Via della Ricerca Scientifica 1, \\ 00133 Rome, Italy \\ ${ }^{4}$ Department of Physics and Department of Mathematics and Computer Science, and J. M. Burgerscentrum \\ and International Collaboration for Turbulence Research, Eindhoven University of Technology, \\ 5600 MB Eindhoven, The Netherlands; CNR-IAC, Via dei Taurini 19, 00185 Rome, Italy; \\ and INFN, Ferrara, Via G. Saragat 1, 44100 Ferrara, Italy \\ ${ }^{5}$ Dipartimento di Fisica, Università di Ferrara and INFN, Ferrara, Via G. Saragat 1, 44100 Ferrara, Italy
}

(Received 1 July 2010; accepted 20 October 2010; published online 23 November 2010)

\begin{abstract}
We present the results of a high resolution numerical study of two-dimensional (2D) RayleighTaylor turbulence using a recently proposed thermal lattice Boltzmann method. The goal of our study is both methodological and physical. We assess merits and limitations concerning small- and large-scale resolution/accuracy of the adopted integration scheme. We discuss quantitatively the requirements needed to keep the method stable and precise enough to simulate stratified and unstratified flows driven by thermal active fluctuations at high Rayleigh and high Reynolds numbers. We present data with spatial resolution up to $4096 \times 10000$ grid points and Rayleigh number up to $\mathrm{Ra} \sim 10^{11}$. The statistical quality of the data allows us to investigate velocity and temperature fluctuations, scale-by-scale, over roughly four decades. We present a detailed quantitative analysis of scaling laws in the viscous, inertial, and integral range, supporting the existence of a Bolgiano-like inertial scaling, as expected in 2D systems. We also discuss the presence of small/large intermittent deviations to the scaling of velocity/temperature fluctuations and the Rayleigh dependency of gradients flatness. () 2010 American Institute of Physics.

[doi:10.1063/1.3517295]
\end{abstract}

\section{INTRODUCTION}

The Rayleigh-Taylor (RT) instability is present whenever we have the superposition of a heavy fluid above a lighter one in a constant acceleration field. ${ }^{1}$ Applications are numerous, from inertial-confinement fusion ${ }^{2}$ to supernovae explosions ${ }^{3}$ and many others. ${ }^{4}$ The RT instability has been studied for decades, but it still presents several open problems. $^{5}$ It is important to control the initial and asymptotic evolution of the mixing layer between two miscible fluids; the small-scale turbulent fluctuations, their anisotropic/isotropic ratio; their dependency on the initial perturbation spectrum, on the geometry of the containing volumes, or on the physical dimensions of the embedding space (see Refs. 6 and 7 for recent high resolution numerical studies). Concerning astrophysical and nuclear applications, the two fluids evolve with strong compressible and/or stratification effects, a situation that is difficult to investigate either theoretically or numerically. The setup studied in this paper is two-dimensional (2D) and the initial configuration is slightly different from what is usually found in literature: the spatial temporal evolution of a single component fluid with a cold uniform region on the top half and a hot uniform region on the bottom half (see Fig. 1 for details). Such a situation is of interest for convection in the atmosphere, ocean, or even stars interiors, where masses of hot/cold fluid may be found in unstable situations. ${ }^{8-10}$ The choice to focus on a $2 \mathrm{D}$ geometry is motivated by different methodological, theoretical, and phenomenological challenges. First, concerning the method, 2D geometries allow to push the numerics up to $4096 \times 10000$ grid points-with correspondingly high Rayleigh/Reynolds numbers; this is an excellent testing ground for the lattice Boltzmann thermal (LBT) scheme $\mathrm{e}^{11,12}$ in fully developed situations, with highly intermittent gradient statistics, and a well developed inertial range of scales with power law distributions. We initially validate the method against exact relationships originating from the hydrodynamical Navier-Stokes-Fourier equations. Then, within the limits settled by the validation steps, we show that the scheme-albeit being only second order accurateallows for quantitative studies of hydrodynamical statistical fluctuations over a four decade interval of scales. From the phenomenological point of view, theoretical works ${ }^{13,14}$ and pioneering numerical simulations ${ }^{15}$ at smaller resolution tell us that Rayleigh-Taylor dynamics in two dimensions displays Bolgiano statistics for velocity and temperature fields, at least at scales small enough and far enough from the edges of the mixing layer. Bolgiano theory, at variance with Kolmogorov theory, ${ }^{16}$ predicts for typical inertial range velocity and temperature fluctuations on a generic inertial scale, $R$, the following laws: 

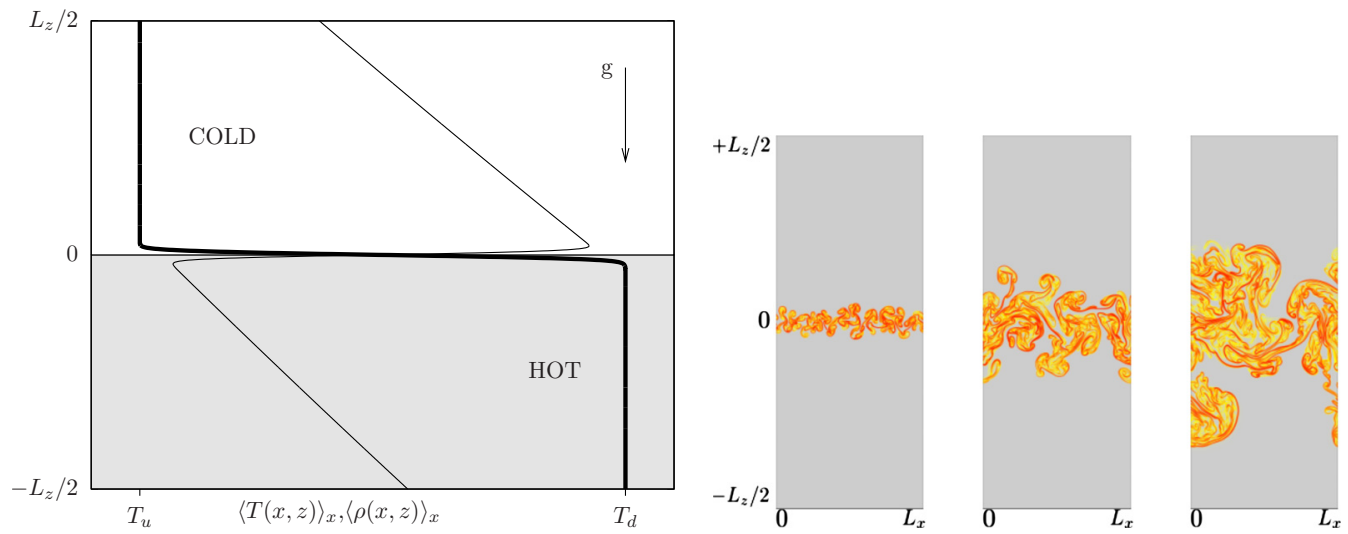

FIG. 1. (Color online) Left: initial configuration for the stratified Rayleigh-Taylor systems. Temperature in the upper half is chosen constant $T_{0}(z)=T_{\text {up }}$, while density follow a hydrostatic profile, $\rho_{0}(z)=\rho_{\text {up }} \exp \left[-g\left(z-z_{c}\right) / T_{\text {up }}\right]$, with $z_{c}$ being the central location in the box. In the lower half, we have $T_{0}(z)=T_{\text {down }}$ and $\rho_{0}(z)=\rho_{\text {down }} \exp \left[-g\left(z-z_{c}\right) / T_{\text {down }}\right]$. To be at equilibrium, we require having the same pressure at the interface, $\rho_{\text {up }} T_{\text {up }}=\rho_{\text {down }} T_{\text {down }}$. The temperature jump at the interface is smoothed by a tanh profile with a width of the order of ten grid points. The bold and tiny solid lines represent the temperature and density profiles, respectively. Right: snapshot of the RT evolution at three times $t=(0.5,1,4) \tau$.

$$
\delta_{R} T \sim\left[\frac{R}{L(t)}\right]^{1 / 5}, \quad \delta_{R} u \sim K(t)\left[\frac{R}{L(t)}\right]^{3 / 5},
$$

where $L(t)$ is a measure of the extension of the mixing layer at any given time $t$ during the RT evolution and $K(t)$ is the square root of the total kinetic energy inside the mixing layer (see below for a precise definition). These scaling properties tell us that temperature/velocity is rougher/smoother than expected for Kolmogorov scaling $\sim R^{1 / 3}$. This is due to the active role played by buoyancy in the vertical momentum evolution, i.e., temperature becomes a fully active scalar at all inertial scales. This is in clear contrast to the Kolmogorov-like phenomenology expected ${ }^{13}$ and observed $^{7,17}$ in three-dimensional (3D) cases. 2D RayleighTaylor systems realize one of those cases where the forcing mechanism-buoyancy-overwhelms nonlinear energy transfer. This has also theoretical relevance, connected to the universality of small-scale statistics in the presence of multiscale forcing mechanisms, ${ }^{18-22}$ in general, or to renormalization group approaches, ${ }^{23}$ in particular. At variance with stochastic external multiscale forcing mechanisms, here the statistics of the buoyancy is directly connected to the velocity field itself, opening the way for new phenomena, which we discuss in detail later. Far from being interesting only for theoretical reasons, Bolgiano scaling is believed to characterize small-scale velocity and temperature fluctuations in 3D Rayleigh-Bénard convection close to the rigid boundaries, where the viscous and thermal boundary layers merge with the bulk region. ${ }^{24}$ In fact, thermohydrodynamical evolution in the proximity of the boundaries is considered to be the key ingredient driving the whole cell behavior. ${ }^{25}$

Here, we will be mainly interested in small-scale properties even though large-scale evolution presents many important open issues, in particular, for stratified flows. For example, we have recently shown that RT evolution in the setup of Fig. 1 is stopped by the adiabatic gradient in the presence of a strongly stratified atmosphere. ${ }^{11}$ The investigation of small-scale properties of such situation, as well as the overshooting observed at the edge of the mixing layer, is in progress and will be reported elsewhere.
All simulations are performed using an innovative LBT, proposed in Ref. 12 and already validated concerning largescale properties on the same geometry here investigated. ${ }^{11}$ Stable, accurate, and efficient discrete kinetic methods describing simultaneous hydrodynamical evolution of momentum and internal energy are notoriously difficult to achieve. $^{26,27}$ The main difficulties stem from the development of subtle instabilities when the velocity increases locally. In recent years, the situation has started to improve, as different attempts have been made to describe active thermal modes within a fully discretized Boltzmann approach. ${ }^{28-35}$

The advantages offered by LB codes are threefold. First, the hydrodynamical manifold is described by the whole Navier-Stokes-Fourier equations, without the need to rely on incompressible and Boussinesq-like approximations. Second, the method is particularly efficient in dealing with complex bulk or boundary physics, opening the way to incorporate either surface tension effects or complex boundary conditions. Last but not least, pressure fluctuations are fully incorporated in the hydrodynamical evolution, so we do not need to solve for Poisson equations; the method becomes fully local in space, allowing for efficient implementations on massively parallel machines even if limited interconnection is available. Building upon this point, our numerical results have been obtained on the QPACE (QCD Parallel Computing on the Cell Broadband Engine) system, a massively parallel machine that uses PowerXCell 8i processors connected by a toroidal network, ${ }^{36,37}$ following the lines of similar older attempts. ${ }^{38}$

The results are as follows. In Sec. II, we present the notation and the main physical quantities that we study in this paper, including a cursory overview of RT large-scale properties. In Sec. III, we briefly summarize the LBT method, we present the numerical details, and we discuss the validation steps. In Sec. IV, we present our results on statistical fluctuations of temperature, velocity, temperature fluxes, and buoyancy terms over the whole range of scales accessed by our numerics. We show that velocity statistics is Bolgiano-like with very small-if any-intermittent correc- 
tions. We discuss the possible origin of these small anomalous corrections, in relation to the corresponding small intermittent fluctuations of the buoyancy term, a new scenario for 2D turbulence. On the other hand, we show that temperature fluctuations are strongly intermittent with high order moments fully dominated by hot/cold fronts. Such strong intermittency has a direct influence also on the temperature flux statistics. Our resolution allows us to address quantitatively and scale-by-scale the statistical properties of all hydrodynamical fields; this analysis is still not within reach in the 3D case. Our concluding remarks (Sec. V) discuss possible further development toward the study of (i) reactive RayleighTaylor systems, (ii) strongly stratified systems, and (iii) multiphase/multicomponent Rayleigh-Taylor or convection systems.

\section{RAYLEIGH-TAYLOR SYSTEMS}

The spatiotemporal evolution of a stratified compressible flow, in an external gravity field, $g>0$, is ruled by the Navier-Stokes-Fourier equations (double indices are meant summed upon)

$$
\left\{\begin{array}{l}
D_{t} \rho=-\rho \partial_{i} u_{i} \\
\rho D_{t} u_{i}=-\partial_{i} P-\rho g \delta_{i, z}+\mu \partial_{j j} u_{i} \\
\rho c_{p} D_{t} T-D_{t} P=\chi \partial_{i i} T,
\end{array}\right.
$$

where $D_{t}$ is the material derivative, $\mu$ and $\chi$ are the molecular viscosity and thermal conductivity, $c_{p}$ is the specific heat at constant pressure, and $\rho, T, P$, and $\boldsymbol{u}$ are density, temperature, pressure, and velocity field, respectively. Under the assumption that compressibility and stratification are small (the situation addressed in this paper) and that fluid parameters depend weakly on the local thermodynamic fields, one can expand pressure around its hydrostatic value $P=P_{0}+p$, with $\partial_{z} P_{0}=-g \rho$ and $p \ll P_{0}$, and perform a small Mach number expansion, ${ }^{39,40}$

$$
\left\{\begin{array}{l}
D_{t} u_{i}=-\frac{\partial_{i} p}{\rho}+\frac{g \theta}{T_{m}} \delta_{i, z}+\nu \partial_{j j} u_{i} \\
D_{t} T-u_{z} \gamma=k \partial_{i i} T .
\end{array}\right.
$$

In this approximation, only temperature fluctuations $\theta$ force the system; we have introduced the mean temperature, $T_{m}$; kinematic viscosity, $\nu=\mu / \rho$; thermal diffusivity, $k=\chi /\left(c_{p} \rho\right)$, and adiabatic gradient for an ideal gas, $\gamma=g / c_{p}$. The small Mach expansion and small stratification decouple the pressure from the internal energy equation, i.e., $p$ in Eq. (3) is just a Lagrange multiplier used to enforce $\partial_{i} u_{i}=0$ everywhere. As we will show in Sec. III, the LBT algorithm we are going to use is meant to reproduce the set of Eqs. (2) and (3) in the corresponding limit.

If the adiabatic gradient is negligible, $\gamma \sim 0$, it is well known that starting from an unstable initial condition, as depicted in Fig. 1, any small perturbation will lead to a turbulent mixture between the hot and the cold regions, expanding along the vertical $z$-direction. Concerning large-scale quantities, a huge amount of earlier work (e.g., see Ref. 5) has focused on the estimation of the growth rate of the mixing layer extension, $L(t)$, and of the total turbulent kinetic energy, $K^{2}(t)=\left[0.5 / L_{x} L(t)\right] \int d x d z u^{2}$, produced by the conversion of the initial potential energy. Using dimensional analysis and self-similar assumptions, ${ }^{41,42}$ one predicts

$$
L(t) \sim \alpha\left(t+t_{0}\right)^{2}, \quad K(t) \sim \beta t,
$$

where $t_{0}$ is the typical time needed for the system to reach a fully nonlinear evolution. The values of the coefficients, $\alpha, \beta$, have been extensively studied both in two and three dimensions. ${ }^{5,11,15,41,43-47}$ They depend on the definition of $L(t)$, typically taken either as the region where the mean temperature profile, averaged over the horizontal direction, $\bar{T}(z)=\left(\frac{1}{L_{x}}\right) \int d x T(x, z, t)$, is within a given range, for example: $\bar{T}(z) \in\left[(1+x) T_{\text {up }}:(1-x) T_{\text {down }}\right]$ with $x=0.05$, or as an integral property over the whole temperature distribution,

$$
L(t)=\frac{1}{L_{x}} \int d x d z \Theta\left[\frac{T(x, z, t)-T_{\text {up }}}{T_{\text {down }}-T_{\text {up }}}\right],
$$

with $\Theta[x]=2 x, 0 \leq x \leq 1 / 2$, and $\Theta[x]=2(1-x), 1 / 2 \leq x \leq 1$. Using the estimate (4), one may predict the whole profile evolution, adopting either simple constant eddy viscosity models or more refined Prandtl mixing length theory. ${ }^{48}$ In Fig. 2, we show the growth rate of the mixing layer, the kinetic energy, and the temporal evolution of the temperature profile as an example of typical evolutions of large-scale quantities in our numerics. The agreement with the expected phenomenology is very satisfactory. Notice a systematic small deviation at a large time. This deviation is probably due to a transition induced by the evolving aspect ratio. When the aspect ratio becomes of order 1, important horizontal fluctuations develop in the system, preventing an efficient conversion of potential energy to vertical kinetic energy (inset in the same figure).

In this paper, on the other hand, we focus on small-scale quantities, i.e., velocity, temperature, and flux statistics scaleby-scale. In particular, we focus on the following set of structure functions, based on moments of order $p$ of velocity, temperature, or mixed increments:

$$
\left\{\begin{array}{l}
S_{\theta}^{(p)}(R, t)=\left\langle\left|\delta_{R} \theta\right|^{p}\right\rangle \\
S_{u_{i}}^{(p)}(R, t)=\left\langle\left|\delta_{R} u_{i}\right|^{p}\right\rangle, \quad i=x, z \\
S_{B}^{(p)}(R, t)=\left\langle\left|\delta_{R} \theta\right|\left|\delta_{R} u_{z}\right|^{p}\right\rangle \\
S_{F}^{(p)}(R, t)=\left\langle\left[\left(\delta_{R} \theta\right)^{2}\left|\delta_{R} u_{z}\right|\right]^{p / 3}\right\rangle,
\end{array}\right.
$$

where we define the increment of a generic hydrodynamical field, $A(x, z, t)$, as $\delta_{R} A=A(x+R, z, t)-A(x, z, t)$ and the average

$$
\langle(\cdot)\rangle=\frac{1}{L_{x} \times L_{z}} \int_{0}^{L_{x}} d x \int_{-\tilde{L}_{z} / 2}^{\tilde{L}_{z} / 2} d z(\cdot)
$$

is performed on the whole horizontal direction and on a given vertical range inside the mixing layer. In order to minimize nonhomogeneous contributions, we typically restrict the vertical extension of the averaging region to $\widetilde{L}_{z}=\frac{1}{2} L(t)$, with $L(t)$ estimated according to the volume average (5). Moreover, in the correlation functions defined above, we only show the results for spatial increments along the fully homogeneous horizontal direction, $\hat{x}$. Subscripts $(B)$ and $(F)$ 
in the third and fourth rows of Eq. (6) denote the correlation functions driving the time evolution of the $p$ th moment of velocity increments (the buoyancy forcing term) and of the temperature flux, respectively. Chertkov ${ }^{13}$ developed a coherent phenomenology for small-scale 2D Rayleigh-Taylor systems on the reasonable assumptions that (i) the mixing layer evolution is adiabatically slow compared to small-scale fluctuations, (ii) the amount of kinetic energy dissipation at small scales is negligible (absence of direct energy cascade in 2D turbulence), and (iii) temperature is efficiently dissipated at small scales (direct temperature cascade). These three ingredients lead to a unique possible dimensional prediction, the Bolgiano scaling (1). In particular, one expects in the inertial range:

$$
\left\{\begin{array}{l}
S_{\theta}^{(p)}(R, t) \sim\left[\frac{R}{L(t)}\right]^{\zeta_{\theta}(p)} \\
S_{u_{x} u_{z}}^{(p)}(R, t) \sim K^{p}(t)\left[\frac{R}{L(t)}\right]^{\zeta_{u}(p)} \\
S_{B}^{(p)}(R, t) \sim K^{p}(t)\left[\frac{R}{L(t)}\right]^{\zeta_{B}(p)}, \quad \eta(t) \ll R \ll L(t) \\
S_{F}^{(p)}(R, t) \sim K^{p / 3}(t)\left[\frac{R}{L(t)}\right]^{\zeta_{F}(p)},
\end{array}\right.
$$

while in the viscous range,

$$
\left\{\begin{array}{l}
S_{\theta}^{(p)}(R, t) \sim\left[\frac{\eta(t)}{L(t)}\right]^{\zeta_{\theta}(p)}\left[\frac{R}{\eta(t)}\right]^{p} \\
S_{u_{x} u_{z}}^{(p)}(R, t) \sim K^{p}(t)\left[\frac{\eta(t)}{L(t)}\right]^{\zeta_{u}(p)}\left[\frac{R}{\eta(t)}\right]^{p} \\
S_{B}^{(p)}(R, t) \sim K^{p}(t)\left[\frac{\eta(t)}{L(t)}\right]^{\zeta_{B}(p)}\left[\frac{R}{\eta(t)}\right]^{p}, \quad R \ll \eta(t) \\
S_{F}^{(p)}(R, t) \sim K^{p / 3}(t)\left[\frac{\eta(t)}{L(t)}\right]^{\zeta_{F}(p)}\left[\frac{R}{\eta(t)}\right]^{p},
\end{array}\right.
$$

with

$$
\zeta_{\theta}(p)=\frac{p}{5}, \quad \zeta_{u}(p)=\frac{3}{5} p
$$

and

$$
\zeta_{B}(p)=\left[\zeta_{\theta}(1)+\zeta_{u}(p)\right], \quad \zeta_{F}(p)=\left[\zeta_{\theta}(2)+\zeta_{u}(1)\right] \frac{p}{3} .
$$

Moreover, according to 2D Bolgiano scaling, the dissipative scale increases with time, as $\eta(t) \sim t^{1 / 8}$. The two expressions [Eqs. (7) and (8)] for inertial and viscous ranges are such that they match at the viscous scale, $\eta(t)$. The presence of a nonstationary evolution makes the problem particularly interesting. The above phenomenology has been already investigated numerically in Ref. 15, where a good agreement with Bolgiano scaling for low order velocity structure functions and a departure from Bolgiano dimensional scaling for temperature structure functions were measured, for the first time. On one hand, the results presented in Ref. 15 clearly indicates the validity of Chertkov's phenomenology, plus the extra complexity of anomalous intermittent corrections to the temperature field. On the other hand, due to limited spatial resolution, the authors of Ref. 15 could not assess statistical properties in a quantitative way scale-by-scale because they had scaling over only about a decade. Our data add to the above discussion a detailed investigation of inertial, viscous, and integral range properties covering all together around four decades. We confirm and measure the presence of large anomalous corrections to the temperature scaling,

$$
\zeta_{\theta}(p)=p / 5+\Delta_{\theta}(p) .
$$

We also show that our data cannot exclude the presence of small deviations from Bolgiano scaling also for velocity field, a novel observation, never reported before and somehow surprising for $2 \mathrm{D}$ turbulence,

$$
\zeta_{u}(p)=3 p / 5+\Delta_{u}(p) .
$$

\section{NUMERICAL METHOD AND VALIDATION STEPS}

\section{A. The thermal lattice Boltzmann algorithm}

In this section, we recall the essential features of the computational lattice Boltzmann method employed in the numerical simulations. A complete analysis, along with extensive validation steps, can be found in Refs. 11 and 12. The thermal-kinetic description of a compressible gas/fluid with variable density $\rho$, local velocity $\boldsymbol{u}$, internal energy $\mathcal{K}$, and subject to a local body force density $\boldsymbol{g}$ is given by the following equations:

$$
\left\{\begin{array}{l}
\partial_{t} \rho+\partial_{i}\left(\rho u_{i}\right)=0 \\
\partial_{t}\left(\rho u_{k}\right)+\partial_{i}\left(P_{i k}\right)=\rho g_{k} \\
\partial_{t} \mathcal{K}+\frac{1}{2} \partial_{i} q_{i}=\rho g_{i} u_{i},
\end{array}\right.
$$

where $P_{i k}$ and $q_{i}$ are the momentum and energy fluxes, still unclosed at this level of description. A recent paper ${ }^{12}$ has shown that it is possible to recover exactly Eq. (11), starting from a suitable discrete version of the Boltzmann equations with self-consistent local equilibria. The reference scheme is summarized by the following set of equation:

$$
f_{l}\left(\boldsymbol{x}+\boldsymbol{c}_{l} \Delta t, t+\Delta t\right)-f_{l}(\boldsymbol{x}, t)=-\frac{\Delta t}{\tau_{\mathrm{LB}}}\left[f_{l}(\boldsymbol{x}, t)-f_{l}^{(\mathrm{eq})}(\boldsymbol{x}, t)\right],
$$

where $f_{l}(\boldsymbol{x}, t)$ represents a probability density function to find a particle at space-time location $(\boldsymbol{x}, t)$ whose velocity $\boldsymbol{c}_{l}$ belongs to a discrete set. ${ }^{33,34}$ The left-hand side of Eq. (12) stands for the streaming step of such probability, whereas the right-hand side represents the relaxation toward local Maxwellian distribution function $f_{l}^{(\text {eq })}$ with characteristic time $\tau_{\mathrm{LB}}$.

The macroscopic fields (density, momentum, and temperature) are defined in terms of the lattice Boltzmann populations,

$$
\rho=\sum_{l} f_{l}, \quad \rho \boldsymbol{u}=\sum_{l} \boldsymbol{c}_{l} f_{l}, \quad D \rho T=\sum_{l}\left|\boldsymbol{c}_{l}-\boldsymbol{u}\right|^{2} f_{l},
$$

with $D$ being the space dimensionality. The novelty of the algorithm employed here stems from the form of the equilib- 
(b)

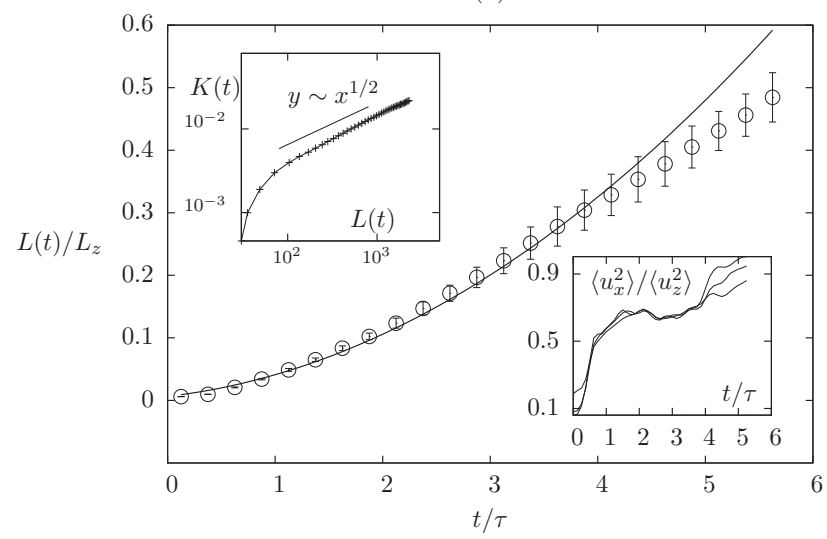

(a)

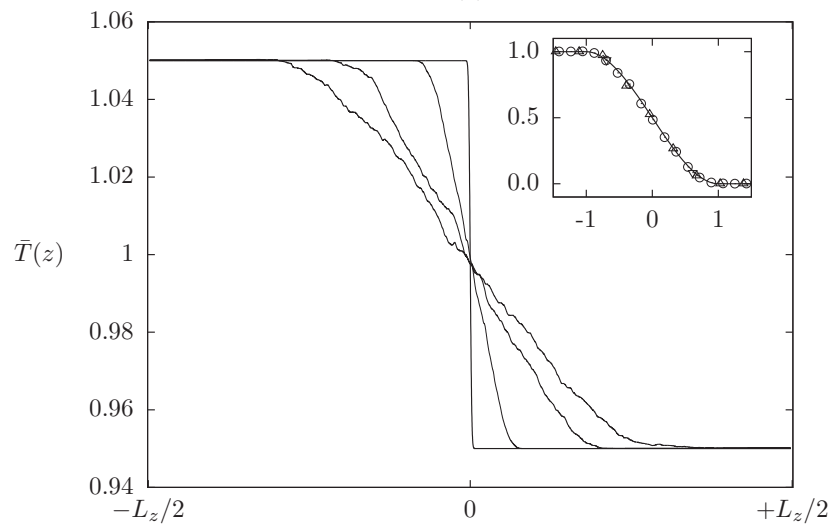

FIG. 2. (a) Mean temperature profile at four different times during the RT evolution. In the inset, we show the rescaling according to the instantaneous mixing layer length $L(t),\left\{\bar{T}[z / L(t), t]-T_{\text {up }}\right\} /\left(T_{\text {down }}-T_{\text {up }}\right)$. The profile rescales perfectly and in agreement with the cubic shape predicted by a Prandtl mixing length theory (Ref. 17) (solid line). (b) Evolution of the mixing layer, $L(t)$, superposed with the best parabolic fit (solid line), using the self-similar prediction (4). Lower inset: ratio between horizontal $\left\langle u_{x}^{2}\right\rangle$ and vertical $\left\langle u_{z}^{2}\right\rangle$ kinetic energy, calculated in the whole, half, or one-quarter of the mixing layer: a transition around $\tau \sim 4$ is clearly visible. Despite this slowing down, the relative scaling of the total kinetic energy with respect to the mixing layer length satisfies the scaling (4). This is shown in the upper inset where we have $K(t) \sim L^{1 / 2}(t)$.

rium distribution function. Here, it directly depends on the coarse grained variables plus a shift from the local body force term,

$$
f_{l}^{(\mathrm{eq})}=f_{l}^{(\mathrm{eq})}\left[\rho, \boldsymbol{u}+\tau_{\mathrm{LB}} \boldsymbol{g}, T+\frac{\tau_{\mathrm{LB}}\left(\Delta t-\tau_{\mathrm{LB}}\right)}{D} g^{2}\right] .
$$

The detailed structure of this equilibrium distribution function can be found in Refs. 11, 33, and 34. Lattice discretization also induces corrections terms in the macroscopic evolution of averaged quantities: both momentum and temperature must be renormalized by discretization effects in order to recover the correct hydrodynamical description from the discretized lattice Boltzmann variables. The first correction to momentum is given by a pre- and postcollisional average, ${ }^{11,49,50}$

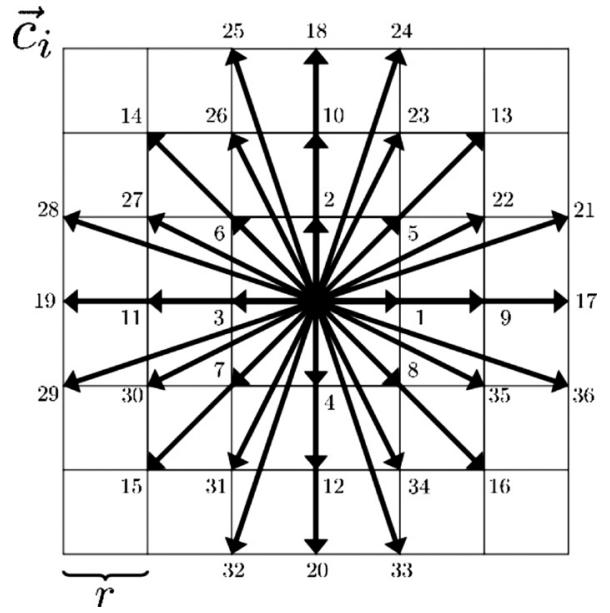

FIG. 3. Scheme of the discrete set of velocities, $r$ is the lattice constant whose value is $r \approx 1.1969$ (Refs. 33 and 34). To recover the correct degree of isotropy for tensors describing thermal fluxes, one needs at least 37 speeds in two dimensions. A smaller set of discrete velocities can be used if off-grid vectors are allowed (Ref. 51).

$$
\boldsymbol{u}^{(H)}=\boldsymbol{u}+\frac{\Delta t}{2} g
$$

and the first nontrivial correction to the temperature field by $^{12}$

$$
T^{(H)}=T+\frac{(\Delta t)^{2} g^{2}}{4 D} .
$$

Using these "renormalized" hydrodynamical fields, it is possible to recover, using a Chapman-Enskog expansion, ${ }^{11,12}$ the standard thermohydrodynamical equations for a compressible fluid with energy conservation. Such procedure, applied to the kinetic Eq. (11), sets the fluxes $P_{i k}$ and $q_{i}$ equal to their hydrodynamical counterpart describing advection, dissipation, and diffusion. In two dimensions $(D=2)$, the resulting equations for the hydrodynamical fields are those given in Eq. (2) (for the explicit calculation, see Ref. 11).

\section{B. Details of the numerical simulations}

We use a 2D LBT algorithm, with 37 population fields (the so-called D2Q37 model), moving in the directions shown in Fig. 3. We have run on the QPACE Supercomputer, ${ }^{36,37}$ a novel massively parallel computer, powered by IBM PowerXCell 8 i processors (an enhanced version of the Cell processor) that supports our algorithm very efficiently. ${ }^{52}$ Three different sets of runs have been performed (parameters are summarized in Table I) at varying accuracy: (A) a fully resolved high resolution simulation, up to $4096 \times 10000$ collocation points with kinematic viscosity and thermal conductivity large enough to ensure optimal resolution of velocity and temperature fields even for large order statistics; (B) a less resolved high resolution simulation, up to $4096 \times 6000$ collocation points, with small-scale transport parameters a factor 2 smaller than in case (A); and (C) an even less resolved case with the same resolution of (B) and viscosity a factor 5 smaller than (A). Runs (B) and (C) make the Rayleigh and Reynolds numbers as large as pos- 
TABLE I. Parameters for the three types of RT runs. Atwood number At $=\left(T_{d}-T_{u}\right) /\left(T_{d}+T_{u}\right)$, viscosity $\nu$; thermal diffusivity $k$; gravity $g$; temperature in the upper half region $T_{u}$; temperature in the lower half region $T_{d}$; normalization time $\tau=\sqrt{L_{x} /(\mathrm{g} \mathrm{At})}$; adiabatic length corresponding to the adiabatic gradient $L_{\gamma}=\Delta T / \gamma$; dissipative scale calculated at $t=\tau, \eta(\tau)$; maximum Rayleigh number $\mathrm{Ra}_{\max }$; and number of independent RT evolution $N_{\text {conf }}$

\begin{tabular}{|c|c|c|c|c|c|c|c|c|c|c|c|c|c|}
\hline & At & $L_{x}$ & $L_{z}$ & $\nu$ & $k$ & $g$ & $T_{\text {up }}$ & $T_{\text {down }}$ & $\tau$ & $L_{\gamma}$ & $\eta(\tau)$ & $\mathrm{Ra}_{\max }$ & $N_{\text {conf }}$ \\
\hline Run (A) & 0.05 & 4096 & 10000 & 0.005 & 0.005 & $2 \times 10^{-5}$ & 0.95 & 1.05 & $6.4 \times 10^{4}$ & 10000 & 4.3 & $8 \times 10^{9}$ & 18 \\
\hline Run (B) & 0.05 & 4096 & 6000 & 0.0025 & 0.0025 & $2.67 \times 10^{-5}$ & 0.95 & 1.05 & $5.5 \times 10^{4}$ & 7500 & 2.2 & $2 \times 10^{10}$ & 5 \\
\hline Run (C) & 0.05 & 4096 & 6000 & 0.001 & 0.001 & $2.67 \times 10^{-5}$ & 0.95 & 1.05 & $5.5 \times 10^{4}$ & 7500 & 1.5 & $1 \times 10^{11}$ & 23 \\
\hline
\end{tabular}

sible even though the statistical properties for subviscous scales will not be as accurate as for set (A). The remarkable result that we are able to present is that the LBT method is able to reproduce large-scale and inertial range physics correctly even in those cases [e.g., runs (B) and (C)], where very small scales are not resolved correctly. A systematic way to validate the accuracy of the method and its convergence toward the hydrodynamical manifold of the kinetic equations is to benchmark the numerical results against the exact relationships coming from the hydrodynamical Navier-Stokes equations of motions. For example, large- and small-scale accuracy can be checked via the equations for the kinetic energy and the enstrophy of the systems,

$$
\left\{\begin{array}{l}
\partial_{t} \frac{1}{2}\left\langle u^{2}\right\rangle_{V}=-\epsilon_{\nu}+g\left\langle\theta u_{z}\right\rangle_{V} \\
\partial_{t} \frac{1}{2}\left\langle w^{2}\right\rangle_{V}=-\epsilon_{\omega}+g\left\langle\partial_{x} \theta w\right\rangle_{V},
\end{array}\right.
$$

where the two dissipative terms are $\epsilon_{\nu}=\nu\left\langle\left(\partial_{i} u_{j}\right)^{2}\right\rangle_{V}$ and $\epsilon_{\nu}=\nu\left\langle w^{2}\right\rangle_{V}$, and with $\langle(\cdot)\rangle_{V}$, we mean the average over the whole volume, and with $w$ the vorticity. These two exact relations probe large and small scales, respectively. In Fig. 4, we show the percentage difference between the left-hand side and the right-hand side normalized with the buoyancy term, for the three set of runs of Table I. Gradients of each field have been calculated either as a centered difference of the hydrodynamical variable or using the lattice definition

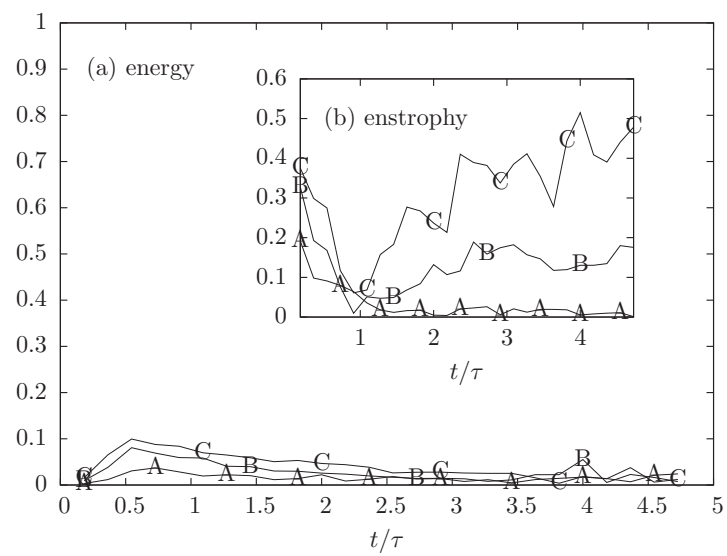

FIG. 4. Large- and small-scale validation of the LBT scheme for the three sets of runs (A)-(C) in Table I. (a) Difference between the left-hand side and the right-hand side of the energy equation in Eq. (15), normalized with the buoyancy term. (b) The same as in (a), but for the enstrophy equation.

$$
\partial_{i} A(x) \approx \sum_{l} \omega_{l} c_{l}^{i} A\left(x+c_{l} \Delta t\right)
$$

with $\omega_{l}$ being the suitable weights ${ }^{11}$ and $\boldsymbol{c}_{l}$ being the lattice velocities (see Fig. 3); we find that the second choice gives better agreement. While the energy balance equation is well verified within a few percent for all resolutions, the enstrophy balance for runs (B) and (C) is not satisfactory. As a result, gradient statistics will be measured only using data from run (A). The next question concerns the range of scales at which accuracy becomes acceptable also for runs (B) and (C). This can be monitored by plotting a sort of normalized "effective gradient" at different scales. In Fig. 5, we show for temperature and vertical velocity the quantities $\tilde{S}_{u_{u}}^{(2)}(R, t)=[L(t) / \eta(t)]^{\zeta u^{(2)}} S_{u_{-}}^{(2)}(R, t) /[K(t) R / \eta(t)]^{2} \quad$ and $\tilde{S}_{\theta}^{(2)}(R, t)=[L(t) / \eta(t)]^{\zeta_{\theta}(2)} S_{\theta}^{(\tilde{2})}(R, t) /[R / \eta(t)]^{2}$ at different

(a)

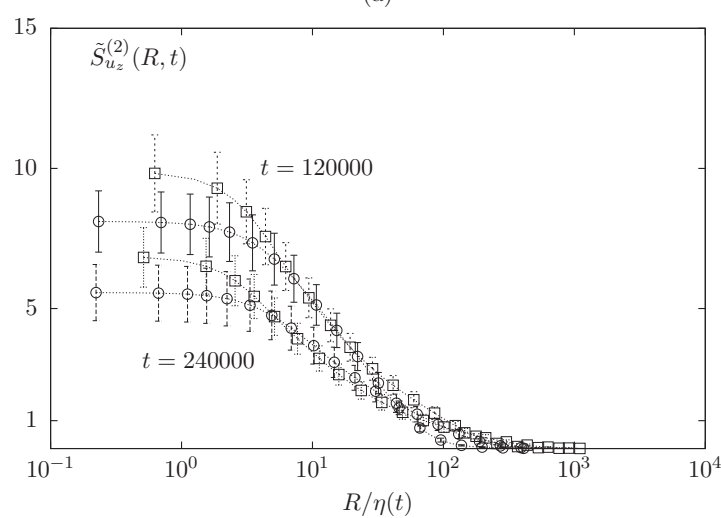

(b)

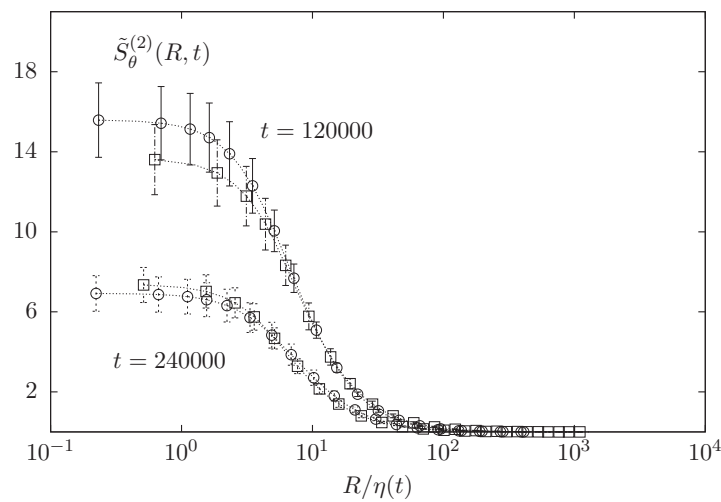

FIG. 5. Normalized effective gradients for runs $(A)(O)$ and $(C)(\square)$ at two different times along the RT evolution: $t=(120000,240000)$ in LBT units. 
times during the RT evolution. Clearly, even though run (C) does not resolve gradients correctly, i.e., the curves do not reach a well developed plateau for small scales, they superpose well with the well resolved run (A) as soon as $R \sim 5 \eta(t)$. This result is important and makes us confident that the LBT numerics is quantitatively accurate even when small scales are not perfectly smooth, i.e., the method provides for a sort of implicit large eddy simulation with an effective subgrid dissipation.

\section{SMALL-SCALE STATISTICS}

As the mixing layer evolves, the effective Rayleigh number, characterizing the thermal instability inside the layer, grows. In the presence of stratification, the expression for the Rayleigh number is not unique. It is possible to introduce a $z$-dependent Rayleigh number, ${ }^{39}$

$$
\operatorname{Ra}(z, t)=\frac{[g / \bar{T}(z)] L^{4}(t)[(\Delta T / L(t))-\gamma]}{\left[\chi / \bar{\rho}(z) c_{p}\right][\mu / \bar{\rho}(z)]},
$$

where the notation $\overline{(\cdot)}$ indicates averages over the horizontal direction. We follow here a common procedure defining a Rayleigh number based on the middle plane, i.e., $\operatorname{Ra}(t)=\operatorname{Ra}(z=0, t)$. Notice that the presence of stratification appears also through the adiabatic term $\gamma$, i.e., any RT mixing of the kind studied here will be stopped sooner or later once an adiabatic atmosphere is reached. For the case when the adiabatic term is not important, $\Delta T / L(t) \gg \gamma$, the ultimate scaling regime predicted by Kraichnan is expected. In this regime, there is a relationship between the normalized heat flux and the Rayleigh number, $7,15,17,53$

$$
\mathrm{Nu} \sim \mathrm{Ra}^{1 / 2},
$$

where the Nusselt number $(\mathrm{Nu})$ is defined as the total heat flux inside the mixing layer normalized with its conducting value: $\mathrm{Nu}=\left\langle\theta u_{z}\right\rangle /[k \Delta T / L(t)]$. Other important output parameters for the system are kinetic energy dissipation and thermal dissipation, $\epsilon_{\nu}, \epsilon_{\theta}$. In two dimensions, we expect that the normalized $\tilde{\epsilon}_{\nu}=\epsilon_{\nu} /\left[K^{3}(t) / L(t)\right]$ and $\tilde{\epsilon}_{\theta}=\epsilon_{\theta} /\left[(\Delta T)^{2} K(t) / L(t)\right]$ vanish and go to a constant for large Rayleigh (Reynolds), respectively. This is tantamount to predict the existence of a direct cascade of temperature fluctuations and the absence of a kinetic energy dissipation anomaly. The monotonic increase of Rayleigh during the mixing layer evolution allows for a check of the previous predictions. In Fig. 6, we show both the Nusselt versus Rayleigh law, confirming for more than four decades the observation of the ultimate regime and the behavior of $\epsilon_{\nu}$ and $\epsilon_{\theta}$ during the RT evolution. We observe the tendency toward a constant nonvanishing dissipative anomaly for temperature fluctuations, while kinetic energy is becoming smaller and smaller at increasing Rayleigh (Reynolds), as expected.

\section{A. Scale-by-scale statistics}

In Fig. 7, we show a log-log plot of $S_{\theta}^{(p)}(R, t)$ and $S_{u_{z}}^{(p)}(R, t)$ for different orders and different times. We also superpose the inertial range scaling predicted by the dimensional Bolgiano prediction. Even though on a log-log scaling

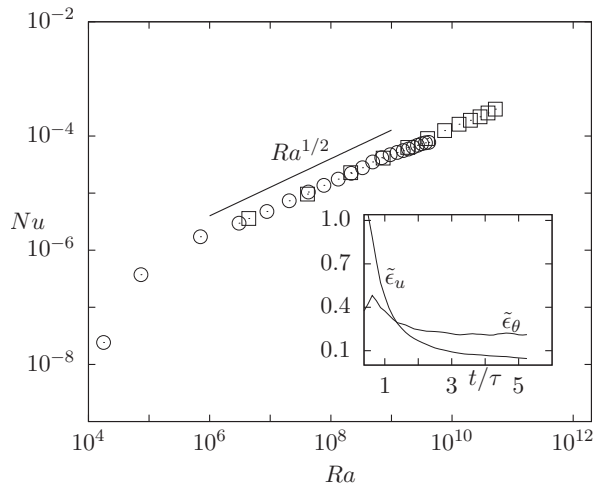

FIG. 6. Nusselt vs Rayleigh, data from run (A) (O) and (C) ( $\square$ ). Inset: dimensionless dissipative anomalies, $\widetilde{\boldsymbol{\epsilon}}_{\nu}$ and $\tilde{\boldsymbol{\epsilon}}_{\theta}$, at changing time during RT evolution.

the global overall agreement between data and dimensional Bolgiano scaling is not bad, important deviations can be seen both at the crossover between viscous and inertial range, $R \sim \eta(t)$, and around the integral scale, $R \sim L(t)$. Let us first investigate the viscous-inertial crossover. There, typical velocity fluctuations have to go from a smooth differentiable behavior $\delta_{R} u \sim R$ to Bolgiano scaling $\delta_{R} u \sim R^{3 / 5}$. The jump in the scaling property is therefore not too large, and one must expect important subleading contributions well inside the inertial range coming from the viscous scaling. Such subleading term may spoil scaling properties even at high Rayleigh values. Differently, for temperature, the jump in the

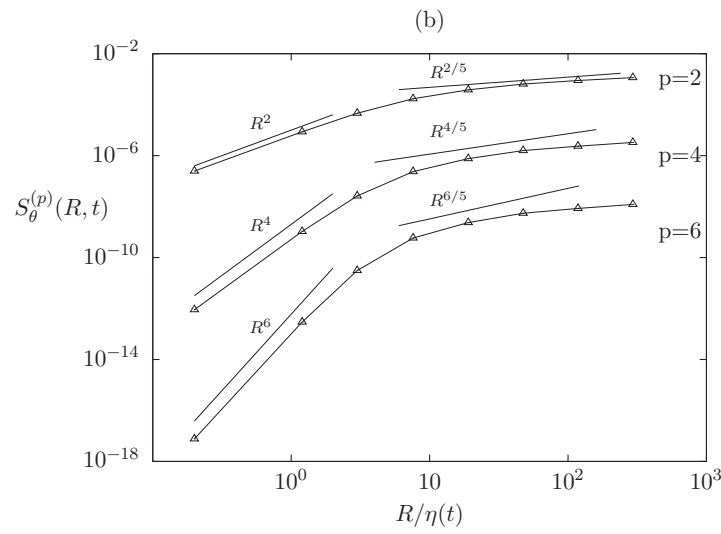

(a)

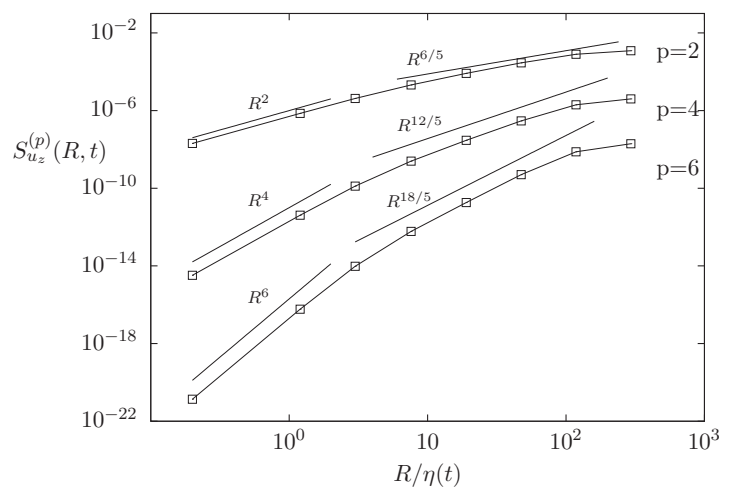

FIG. 7. Log-log plot of velocity (a) and temperature (b) scaling for $p=2,4,6$ at a late time during RT evolution $[t \sim 5 \tau$ and data from run (A) $]$. We also plot the corresponding Bolgiano and viscous scaling. 
scaling properties from viscous to inertial is large (from $R^{p}$ to $R^{p / 5}$ ). Subleading terms cannot play any role. Anyhow, such a big change in the scaling properties cannot happen in a too short range of scales: the interval of increments with neither a pure viscous nor a pure inertial scaling should be large in this case.

A "conveniently simple transition function" to encompass both viscous and inertial range scaling in a single fitting expression is given by the Batchelor parametrization, ${ }^{54-58}$ which for a generic structure function of order $p$ reads as

$$
F^{(p)}(R, t)=C_{p} \frac{R^{p}}{\left[R^{2}+A_{p} \eta^{2}(t)\right]^{[p-\zeta(p)] / 2}},
$$

where $C_{p}$ is a suitable dimensional normalization parameter and $A_{p}$ is a dimensionless parameter taking into account some small possible dependency of the viscous cutoff on the order of the correlation function. ${ }^{59-62}$ The above expression is the simplest way to glue smoothly a differential behavior in the viscous range, $\sim R^{p}$, for $R \ll \eta(t)$ with a rough scaling, $\sim R^{\zeta(p)}$, in the inertial range, $\eta(t) \ll R$. We need also to match the inertial-integral layer, $R \sim L(t)$, where structure functions start to saturate because all hydrodynamical fields decorrelate for $R \gg L(t)$. It is easy to generalize the Batchelor parametrization to also encompass such a region of scales, reaching a global phenomenological description of structure functions valid for all scales,

$$
F^{(p)}(R, t)=C_{p} \frac{R^{p}}{\left[R^{2}+A_{p} \eta^{2}(t)\right]^{[p-\zeta(p)] / 2}}\left[R^{a}+B L^{\alpha}(t)\right]^{-\zeta(p) / a},
$$

where in the above expression, the crossover around $R \sim L(t)$ is fixed by the parameters $a$ and $B$ (in the following, always chosen as $a=4$ and $B=1$ ). The potentialities of the parametrization (18) cannot be appreciated on log-log plots: a detailed scale-by-scale analysis of structure functions behavior is needed.

A scale-by-scale analysis can be obtained by looking at the so-called local scaling exponents (LSEs), i.e., the log derivatives of any structure function,

$$
\zeta(p \mid R, t)=\frac{d \log \left[F^{(p)}(R, t)\right]}{d \log (R)} .
$$

Whenever we have a pure power law behavior, the output must be a constant as a function of the separation scale, $R$, $\zeta(p \mid R, t) \sim \zeta(p, t)$. The advantage to measure Eq. (19) stems from the possibility to follow also the crossover between viscous and inertial range and between inertial and integral range, scale-by-scale, hence the name. In Fig. 8, we show for $p=4,6$ the velocity structure functions against the Batchelor parametrization (18) for two different Rayleigh numbers. In the body of the figure, we plot the LSE for $S_{u_{z}}^{(p)}(R, t)$ from our data and superposed with the corresponding expression coming from the parametrization (18), where we have used the Bolgiano value $\zeta_{u}(p)=\frac{3}{5} p$. The agreement is strikingly good; considering together all data at different resolutions, we are able to reproduce the viscous, inertial, and integral scale behavior over four decades of scaling range. The agreement
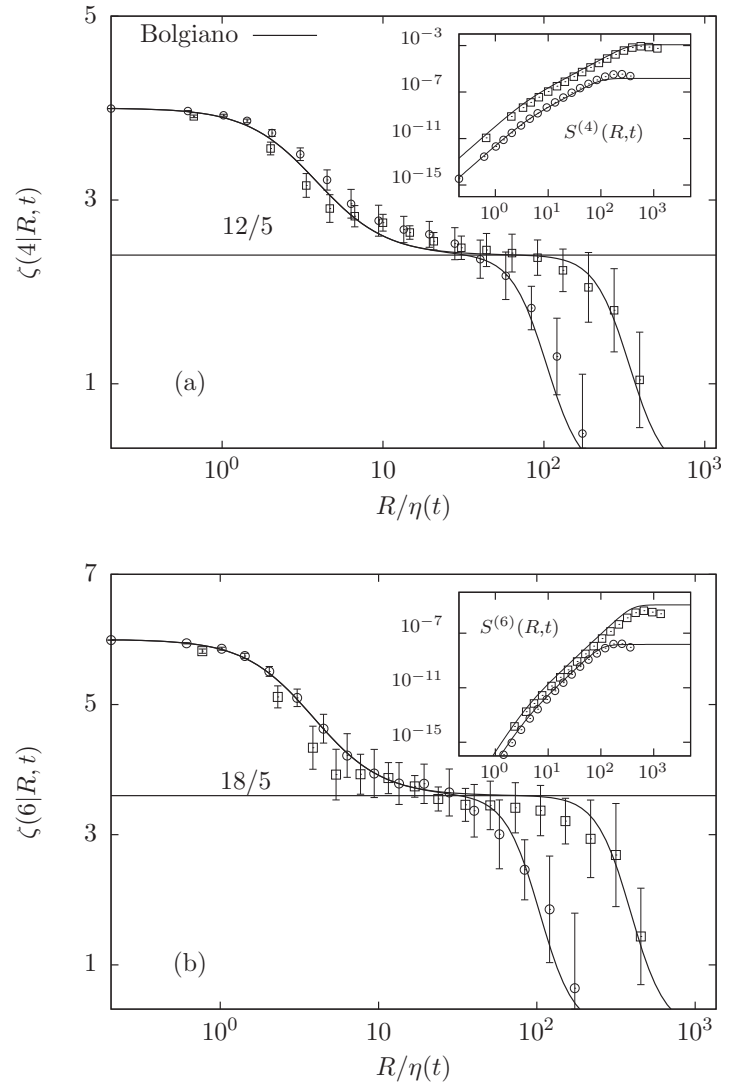

FIG. 8. Local scaling properties for velocity structure functions, $\zeta_{u_{z}}(p \mid R, t)$ for runs (A) (O) at $t=4 \tau$ and (C) ( $\square$ ) at $t=4 \tau$. We show the case with $p=4$ [panel (a)] and $p=6$ [panel (b)]. Solid lines correspond to the local scaling exponents as predicted from Eq. (18) using the Bolgiano dimensional scaling (9), also drawn as a horizontal line with values of 12/5 and $18 / 5$, respectively. Error bars are calculates out of the scattering between the $N_{\text {conf }}$ different RT evolutions for each run. Insets: structure functions, $S_{u_{z}}^{(p)}(R, t)$, for $p=4$ and $p=6$ and the two runs (A) and (C) (same symbols). The solid line is the parametrization (18).

between the Bolgiano dimensional prediction and the velocity scaling is very accurate within error bars. Notice that the use of LSE with respect to log-log scaling, as depicted in the inset of the same figure, allows to move the discussion from global fit over many orders of magnitude (for the latter) to a scale-by-scale fit of $\mathcal{O}(1)$ quantities (for the former). Moving to temperature scaling, the scenario changes. In Fig. 9, we show the same as Fig. 8 but for temperature and up to $p=8$. Here, the agreement with the Batchelor parametrization with the Bolgiano dimensional scaling for temperature $\zeta_{\theta}(p)=p / 5$, is less good, almost acceptable for low order moments, but definitely not on top of the numerical data for high order moments. In order to achieve a good fit, on the whole range of scale, one needs to introduce anomalous corrections to the exponents $\zeta_{\theta}(p)=p / 5+\Delta_{\theta}(p)$ used in the Batchelor formula. In the same figure, we show indeed how the use of $\Delta_{\theta}(4)=-0.2, \Delta_{\theta}(6)=-0.5$, and $\Delta_{\theta}(8)=-0.8$ gives a much better agreement between the numerical data and the phenomenological parametrization formula (18). This is, in our view, a very clean demonstration of the existence of anomalous scaling for temperature fluctuations in 2D RT. The values measured for $\Delta_{\theta}(p)$ are in agreement with the one 

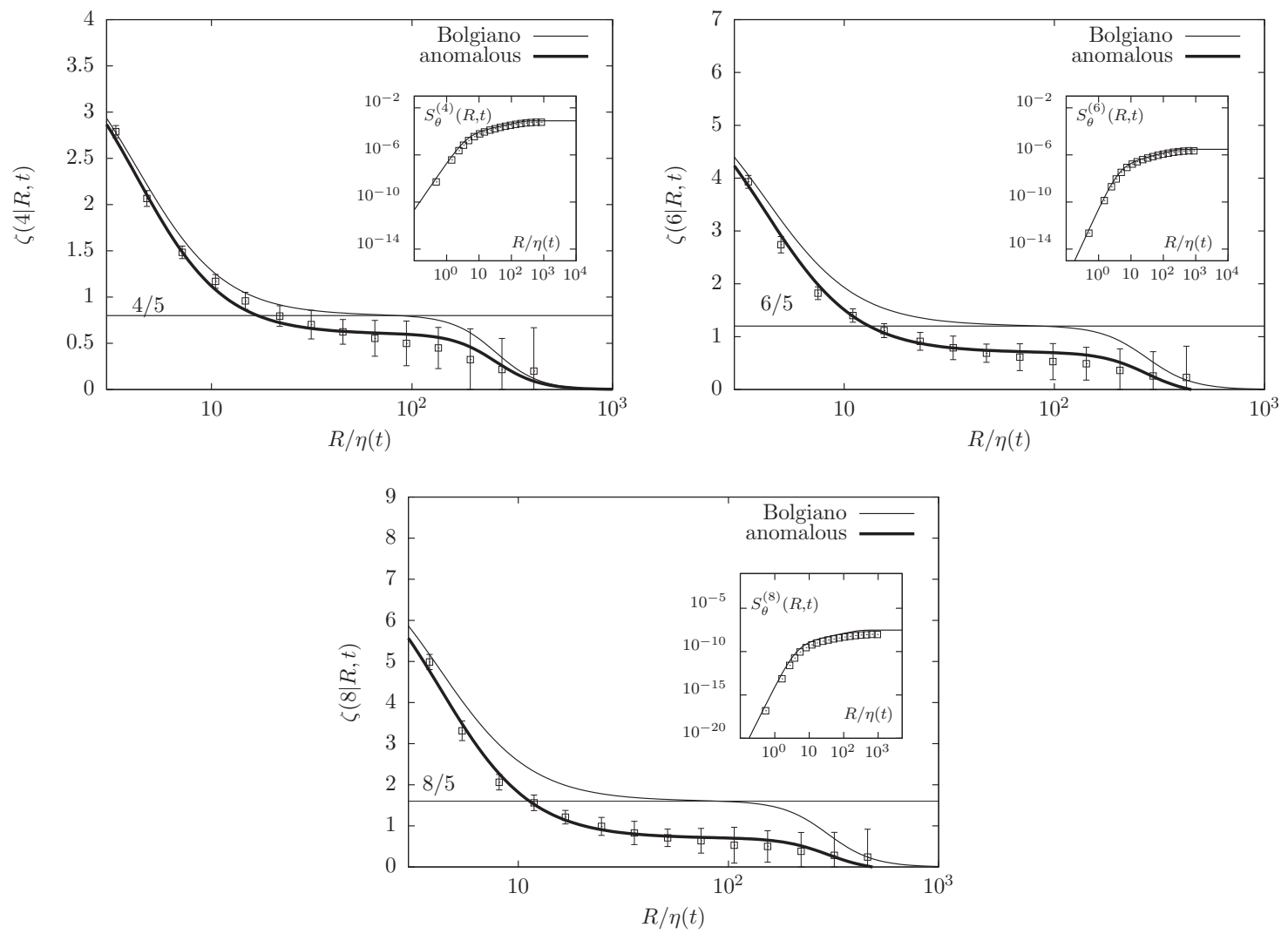

FIG. 9. The same data as in Fig. 8, but for temperature scaling. We show $p=4, p=6$, and $p=8$. The solid line corresponds to the parametrization (18) using the dimensional Bolgiano scaling exponents. The thick solid line is the same parametrization but with anomalous scaling exponents. Already for $p=4$ and more importantly for $p=6$ and $p=8$ the LSE for the parametrization (18) with dimensional Bolgiano scaling $\zeta(p)=p / 5$ does not fit the numerical data. As a guide to the eyes, we also show the Bolgiano inertial range values as horizontal lines in each panel. The curves supporting anomalous scaling are obtained with the following correction to the exponents: $\Delta_{\theta}(4)=-0.2, \Delta_{\theta}(6)=-0.5$, and $\Delta_{\theta}(8)=-0.7$. Insets: structure functions superposed with the Batchelor parametrization with anomalous inertial exponents (solid line).

presented in Ref. 15. The result on temperature scaling are summarized in Table II.

An important feature of RT in two dimensions is the active role played by buoyancy at all scales, as witnessed by the Bolgiano phenomenology. The interesting point here is that as the buoyancy is driven by temperature fluctuations, the forcing mechanism in the momentum equations is given by a non-self-similar-intermittent-field. Navier-Stokes equations forced with power law forcing have attracted the attention in the past both for the application of the renormalization group ${ }^{23}$ and for issues concerning small-scale universality, i.e., understanding how strong must be the forcing mechanism in order to change the small-scale statistics in turbulent flows. ${ }^{18-21}$ Typically, for any given system, there exists a critical exponent $b_{c}$ characterizing the power law decaying of the forcing spectrum, $E(k) \sim k^{-b}$, such that for

TABLE II. Summary of temperature scaling exponents using the best fit obtained by the parametrization (18) using for $\eta(t)$ and $L(t)$ the actual values measured on the data.

\begin{tabular}{lccc}
\hline \hline$\zeta_{\theta}(p)$ & Bolgiano & Ref. 15 & Here \\
\hline$p=4$ & 0.8 & 0.6 & $0.6 \pm 0.06$ \\
$p=6$ & 1.2 & 0.7 & $0.7 \pm 0.07$ \\
$p=8$ & 1.6 & $\cdots$ & $0.8 \pm 0.1$ \\
\hline \hline
\end{tabular}

$b<b_{c}$ the forcing is the leading mechanism of energy exchange at all scales. In our case, the very existence of Bolgiano scaling tells us that we fall in the latter class. The main interesting difference here, with previous theoretical and numerical studies, is that the forcing mechanism is also intermittent, i.e., very different from the typical scaling-invariant Gaussian and delta-correlated in time power law forcing used in Refs. 18, 19, and 23. Indeed, the high intermittency of the temperature scaling shown in Sec. III suggests the possibility that some degree of intermittency is also hidden in the velocity field, even though a direct measure, as the one shown in Fig. 8, rules out large effects. In Fig. 10, we are looking directly at the forcing statistics entering in the equation of high order velocity moments, what we call the buoyancy structure functions in Eq. (6), $S_{B}^{(p)}(R, t)$. As one can see, even there, it is hard to disentangle any deviations from Bolgiano dimensional scaling. A different scenario appears for the temperature flux structure functions, $S_{F}^{(p)}(R, t)$, as defined in Eq. (6), shown in Fig. 11. Here, a deviation from the dimensional scaling is visible due to the higher order of temperature fields with respect to the velocity fields entering in these correlation functions. A possible way to highlight even better intermittent correction is to look at the behavior of velocity and temperature hyperflatness, 


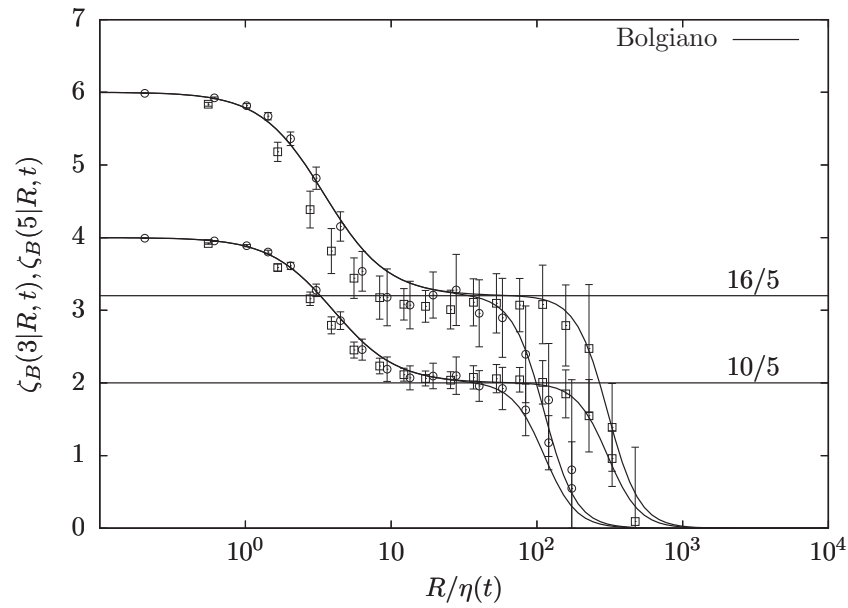

FIG. 10. Local scaling exponent for buoyancy terms, $S_{B}^{(p)}(R, t)$, with $p=1,3$ for runs (A) (O) and (C) $(\square)$. The solid line corresponds to the dimensional estimate (18) with $\zeta_{B}(p)=\zeta_{\theta}(1)+\zeta_{u_{z}}(p)$. The two horizontal lines give the expected Bolgiano scaling in the inertial range, 10/5 $(p=3)$ and $16 / 5(p=5)$.

$$
F_{u_{z}}(R, t)=\frac{S_{u_{z}}^{(4)}(R, t)}{\left[S_{u_{z}}^{(2)}(R, t)\right]^{2}}, \quad F_{\theta}(R, t)=\frac{S_{\theta}^{(4)}(R, t)}{\left[S_{\theta}^{(2)}(R, t)\right]^{2}} .
$$

Any systematic dependence of flatness on the reference scale $R$ is the signature of a nonperfect self-similar statistics. Figure 12 shows temperature and velocity flatness at two different times during the RT evolution. Temperature is clearly intermittent with a flatness, which increases at decreasing scale. Velocity is more noisy; nevertheless, our data cannot exclude a small-scale dependency of flatness also for the latter, pointing toward small but detectable breaking of selfsimilarity, i.e., corrections to the Bolgiano scaling also for velocity. In the inset of the same figure, we show the relative scaling of fourth and sixth order structure functions versus

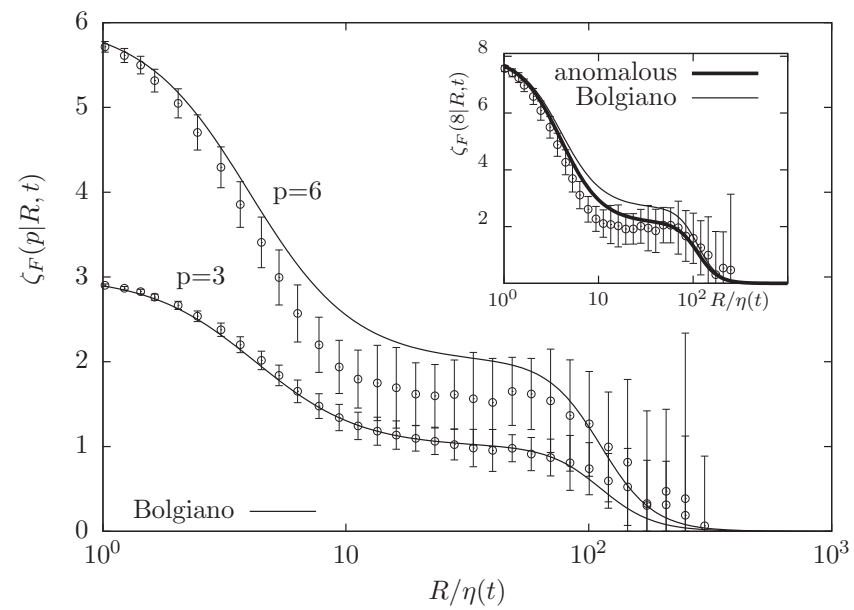

FIG. 11. Run (A): local scaling exponent for temperature flux moments, $S_{F}^{(p)}(R, t)$, with $p=3,6$ and $p=8$ (inset). The solid thin line for $p=3,6$ (main plot) corresponds to the dimensional Bolgiano estimate (18) with $\zeta(p)=\left(\zeta_{\theta}(2)+\zeta_{u_{z}}(2)\right) p / 3$. In the inset, we show fit with both dimensional Bolgiano and anomalous estimates. Notice the better agreement with the anomalous case.
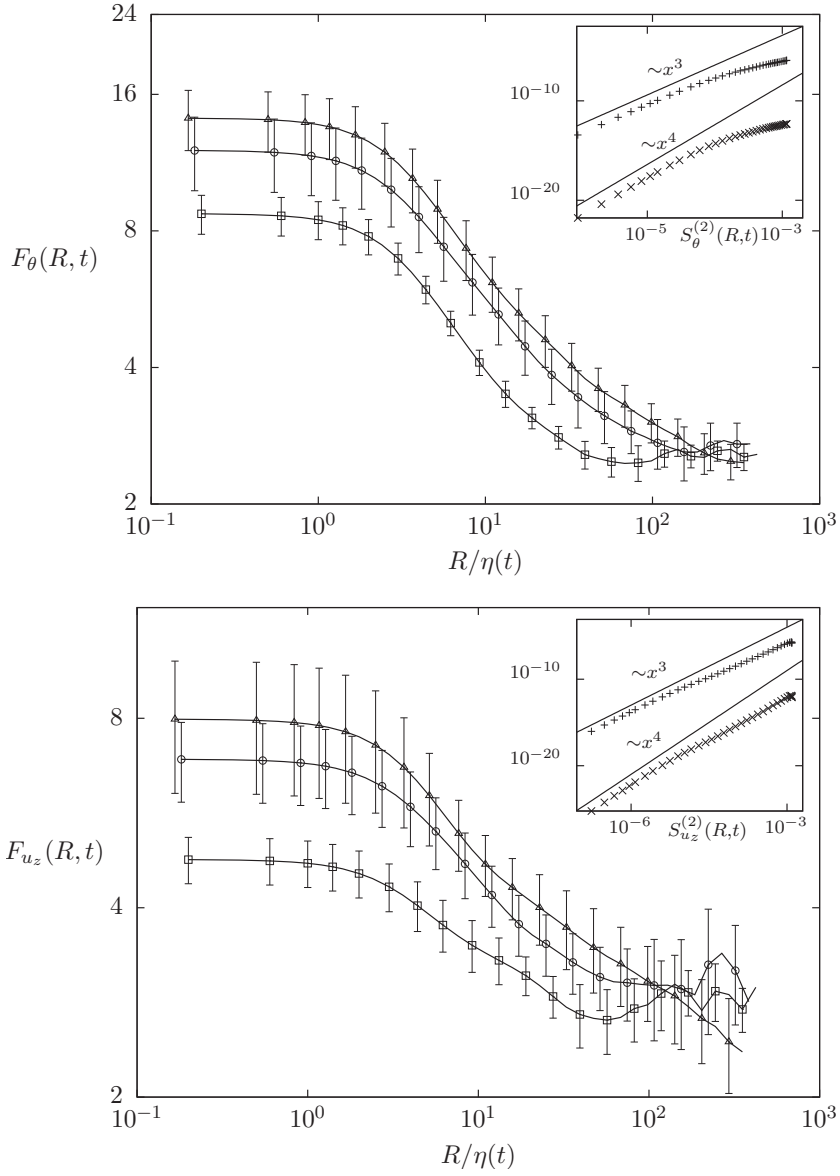

FIG. 12. Velocity (bottom) and temperature (top) flatness at three different times during RT evolution $t=(2,3,5) \tau$ in run (A). Insets: ESS plot for structure functions of order $p=6(+)$ and $p=8(\times)$ vs structure function with $p=2$. The solid line corresponds to the dimensional scaling.

the second order one, a procedure known as ESS (Extended Self Similarity) in literature ${ }^{63,64}$

$$
\begin{aligned}
& S_{\theta}^{(p)}(R, t) \quad \text { versus } S_{\theta}^{(2)}(R, t), \\
& S_{u_{z}}^{(p)}(R, t) \quad \text { versus } S_{u_{z}}^{(2)}(R, t) .
\end{aligned}
$$

Here, a breaking of self-similarity is detected as a deviation from the dimensional scaling $S^{(p)}(R, t)=\left[S^{(2)}(R, t)\right]^{p / 2}$. Deviations for the temperature/velocity are strong/small and clearly detectable.

The above results suggest that in order to highlight some possible nontrivial scaling properties in the velocity statistics, one needs to look at small scales, where temperature intermittency becomes more intense and possibly affects also the momentum equations. In Fig. 13, we show the behavior of the flatness of velocity and temperature derivatives during the RT evolution, i.e., at increasing Rayleigh,

$$
F_{\partial_{x} u_{z}}(t)=\frac{\left\langle\left(\partial_{x} u_{z}\right)^{p}\right\rangle}{\left\langle\left(\partial_{x} u_{z}\right)^{2}\right\rangle^{p / 2}}, \quad F_{\partial_{x} \theta}(t)=\frac{\left\langle\left(\partial_{x} \theta\right)^{p}\right\rangle}{\left\langle\left(\partial_{x} \theta\right)^{2}\right\rangle^{p / 2}} .
$$

Both small-scale temperature and velocity intermittency are increasing, with the temperature case much faster. We fit a power law behavior, 


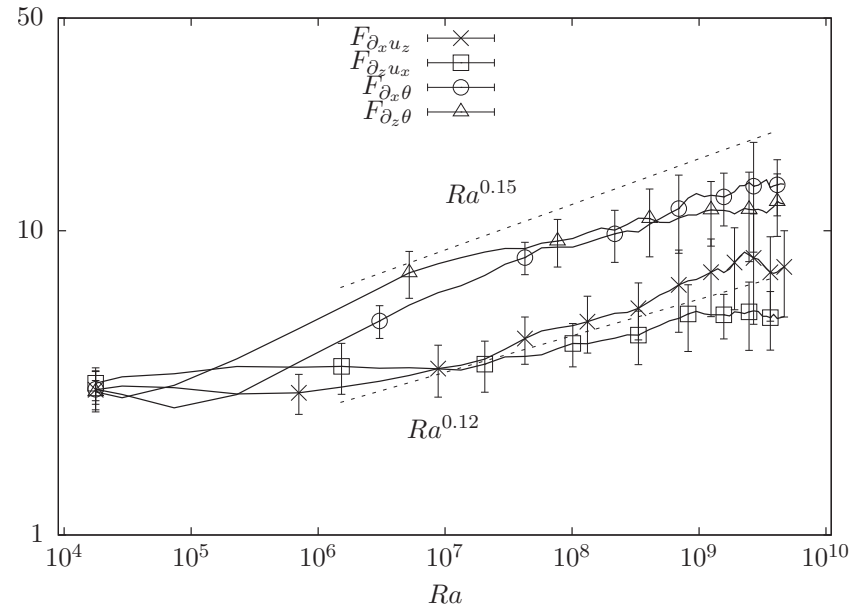

FIG. 13. Flatness based on velocity and temperature gradients as a function of the Rayleigh number. Two power laws with the best fit for $\mathrm{Ra} 10^{7}$ are also shown as a guide to the eyes.

$$
F_{u_{z}}^{(p)} \sim \mathrm{Ra}^{\xi_{\partial_{x}} u_{z}(p)}, \quad F_{\theta}^{(p)} \sim \mathrm{Ra}^{\xi_{\partial_{x}} \theta^{(p)}},
$$

with $\xi_{\partial_{x}} u_{z}(p)=0.12(5)$ and $\xi_{\partial_{x}}(p)=0.15(5)$. While the result for temperature is not surprising, the result for velocity is supporting the existence of a small, but detectable intermittent correction to the 2D Bolgiano scaling for the velocity field. In the same figure, we also measure the temperature and horizontal-velocity flatness along the vertical direction in order to have a quantitative assessment on the importance of residual small-scale anisotropy in the system. ${ }^{65}$ Both measurements coincides with those measured on the horizontal directions, suggesting little, if any, residual anisotropy at those scales.

\section{CONCLUSIONS AND FURTHER DEVELOPMENTS}

In this paper, we have presented the results of a high resolution numerical study of 2D Rayleigh-Taylor turbulence using a new thermal lattice Boltzmann method. The goal of this study was both methodological and physical. Concerning the method, we validate and assess the stability, accuracy, and performances of the numerical discrete kinetic algorithm used, showing that even when not perfectly resolved at small scales, inertial and integral scale hydrodynamics is well reproduced. This result opens the way to a systematic exploitation of LBT algorithms also for fully developed turbulence. Concerning the physics of RT turbulence in two dimensions, we have analyzed data up to $\mathrm{Ra} \sim 10^{11}$ and shown that the dynamics is dominated by a Bolgiano phenomenology, i.e., thermal fluctuations in the buoyancy term are overwhelming the kinetic energy flux at all scales. We have also shown that (i) a suitable Batchelor-like parametrization is able to reproduce scale-by-scale the whole statistics at all scales, over four decades; (ii) temperature fluctuations show small-scale intermittency, with scaling exponents tending to saturate at high orders (see Table II), a signature of persistence of hot/cold fronts even at very small scales; ${ }^{66}$ and (iii) velocity statistics is much closer to Bol- giano dimensional scaling even if small intermittent corrections cannot be ruled out, especially concerning gradients evolution.

All these results are relevant for 3D thermal systems in the presence of boundaries. Indeed, Bolgiano physics is believed to describe also thermal and velocity fluctuations close to the boundary in real 3D convective RayleighBènard cells. ${ }^{67,68}$ The existence of anomalous intermittent small-scale fluctuations also in these cases is relevant to control the physics of the viscous and thermal boundary layers.

The algorithm presented here opens the way for natural generalization to more complex situations. First, it is trivially extendable to 3D cases. Second, it can be further generalized, including bulk forcing terms in the internal energy equation, to describe reactive system. Third, the possibility to couple the thermal LBT scheme with multicomponent and/or multiphase LBT models is under investigation, ${ }^{69}$ including nontrivial wettability properties at the boundaries: ${ }^{70}$ a case of interest to describe convection of boiling systems.

\section{ACKNOWLEDGMENTS}

We acknowledge useful discussions with A. Mazzino, G. Boffetta, A. Celani, and K. Sugiyama. We warmly thank the QPACE development team for the support during the implementation of our code and execution of our simulations. We furthermore acknowledge access to QPACE and eQPACE during the bring-up phase of these systems. Parts of the preliminary simulations were also performed on computing resources made available by CASPUR under HPC Grant 2009.

${ }^{1}$ S. Chandrasekhar, Hydrodynamic and Hydromagnetic Stability (Clarendon, Oxford, 1961).

${ }^{2}$ J. D. Lindl, Inertial Confinement Fusion (Springer-Verlag, New York, 1998).

${ }^{3}$ M. Zingale, S. E. Woosley, C. A. Rendleman, M. S. Day, and J. B. Bell, "Three-dimensional numerical simulations of Rayleigh-Taylor unstable flames in type Ia supernovae," Astrophys. J. 632, 1021 (2005).

${ }^{4}$ D. H. Sharp, "An overview of Rayleigh-Taylor instability," Physica D 12, 3 (1984).

${ }^{5}$ G. Dimonte, D. L. Youngs, A. Dimits, S. Weber, M. Marinak, S. Wunsch, C. Garasi, A. Robinson, M. J. Andrews, P. Ramaprabhu, A. C. Calder, B. Fryxell, J. Biello, L. Dursi, P. MacNeice, K. Olson, P. Ricker, R. Rosner, F. Timmes, H. Tufo, Y.-N. Young, and M. Zingale, "A comparative study of the Rayleigh-Taylor instability using high-resolution three-dimensional numerical simulations: The Alpha Group collaboration," Phys. Fluids 16, 1668 (2004).

${ }^{6}$ D. Livescu, J. R. Ristorcelli, R. A. Gore, S. H. Dean, W. H. Cabot, and A. W. Cook, "High Reynolds numbers Rayleigh-Taylor turbulence," J. Turbul. 10, 13 (2009).

${ }^{7}$ G. Boffetta, A. Mazzino, S. Musacchio, and L. Vozzella, "Kolmogorov scaling and intermittency in Rayleigh-Taylor turbulence," Phys. Rev. E 79, 065301 (2009).

${ }^{8}$ V. M. Canuto and J. Christensen-Dalsgaard, "Turbulence in astrophysics: Stars," Annu. Rev. Fluid Mech. 30, 167 (1998).

${ }^{9}$ P. M. M. Soares, P. M. A. Miranda, A. P. Siebesma, and J. Teixeira, "An eddy-diffusivity/mass-flux parametrization for dry and shallow cumulus convection," Q. J. R. Meteorol. Soc. 130, 3365 (2004).

${ }^{10}$ A. P. Siebesma, P. M. M. Soares, and J. Teixeira, "A combined eddydiffusivity mass-flux approach for the convective boundary layer," J. Atmos. Sci. 64, 1230 (2007).

${ }^{11}$ A. Scagliarini, L. Biferale, M. Sbragaglia, K. Sugiyama, and F. Toschi, "Lattice Boltzmann methods for thermal flows: Continuum limit and applications to compressible Rayleigh Taylor systems," Phys. Fluids 22, 055101 (2010)

${ }^{12}$ M. Sbragaglia, R. Benzi, L. Biferale, H. Chen, X. Shan, and S. Succi, 
"Lattice Boltzmann method with self-consistent thermo-hydrodynamic equilibria,” J. Fluid Mech. 628, 299 (2009).

${ }^{13}$ M. Chertkov, "Phenomenology of Rayleigh-Taylor turbulence," Phys. Rev. Lett. 91, 115001 (2003).

${ }^{14} \mathrm{~N}$. Vladimirova and M. Chertkov, "Self-similarity and universality in Rayleigh Taylor, Boussinesq turbulence," Phys. Fluids 21, 015102 (2009).

${ }^{15}$ A. Celani, A. Mazzino, and L. Vozella, "Rayleigh-Taylor turbulence in two dimensions," Phys. Rev. Lett. 96, 134504 (2006).

${ }^{16}$ U. Frisch, Turbulence: The Legacy of A. N. Kolmogorov (Cambridge University Press, Cambridge, 1995).

${ }^{17}$ G. Boffetta, A. Mazzino, S. Musacchio, and L. Vozella, "Statistics of mixing in three-dimensional Rayleigh-Taylor turbulence at low Atwood number and Prandtl number one," Phys. Fluids 22, 035109 (2010).

${ }^{18}$ A. Sain, Manu, and R. Pandit, "Turbulence and multiscaling in the randomly forced Navier-Stokes equation,” Phys. Rev. Lett. 81, 4377 (1998).

${ }^{19}$ L. Biferale, A. S. Lanotte, and F. Toschi, "Effects of forcing in threedimensional turbulent flows," Phys. Rev. Lett. 92, 094503 (2004).

${ }^{20}$ A. Cheskidov, C. R. Doering, and N. P. Petrov, "Energy dissipation in fractal-forced flow," J. Math. Phys. 48, 065208 (2007).

${ }^{21}$ D. Hurst and J. C. Vassilicos, "Scalings and decay of fractal-generated turbulence," Phys. Fluids 19, 035103 (2007).

${ }^{22}$ L. Biferale, M. Cencini, A. S. Lanotte, M. Sbragaglia, and F. Toschi, "Anomalous scaling and universality in hydrodynamic systems with power-law forcing," New J. Phys. 6, 37 (2004).

${ }^{23}$ A. Mazzino, P. Muratore-Gianneschi, and S. Musacchio, "Scaling regimes of $2 \mathrm{~d}$ turbulence with power-law stirring: Theories versus numerical experiments," J. Stat. Mech.: Theory Exp. 2009, P10012.

${ }^{24}$ D. Lohse and K. Q. Xia, "Small-scale properties of turbulent RayleighBènard convection," Annu. Rev. Fluid Mech. 42, 335 (2010).

${ }^{25}$ G. Ahlers, S. Grossmann, and D. Lohse, "Small-scale properties of turbulent Rayleigh-Bènard convection heat transfer and large-scale dynamics in turbulent Rayleigh-Bènard convection," Rev. Mod. Phys. 81, 503 (2009).

${ }^{26} \mathrm{~S}$. Succi, The Lattice Boltzmann Equation (Oxford Science, New York, 2001).

${ }^{27}$ D. Wolf-Gladrow, Lattice-Gas Cellular Automata and Lattice Boltzmann Models (Springer, New York, 2000).

${ }^{28} \mathrm{P}$. Lallemand and L. S. Luo, "Theory of the lattice Boltzmann method: Acoustic and thermal properties in two and three dimensions," Phys. Rev. E 68, 036706 (2003).

${ }^{29}$ N. I. Prasianakis and I. V. Karlin, "Lattice Boltzmann method for thermal flow simulation on standard lattices," Phys. Rev. E 76, 016702 (2007).

${ }^{30} \mathrm{~V}$. Sofonea, "Implementation of diffuse reflection boundary conditions in a thermal lattice Boltzmann model with flux limiters," J. Comput. Phys. 228, 6107 (2009).

${ }^{31}$ G. Gonnella, A. Lamura, and V. Sofonea, "Lattice Boltzmann simulation of thermal nonideal fluids," Phys. Rev. E 76, 036703 (2007).

${ }^{32}$ M. Watari, "Velocity slip and temperature jump simulations by the threedimensional thermal finite-difference lattice Boltzmann method," Phys. Rev. E 79, 066706 (2009).

${ }^{33}$ P. C. Philippi, L. A. Hegele, L. O. E. dos Santos, and R. Surmas, "From the continuous to the lattice Boltzmann equation: The discretization problem and thermal models," Phys. Rev. E 73, 056702 (2006).

${ }^{34}$ X. Shan, F. Yuan, and H. Chen, "Kinetic theory representation of hydrodynamics: A way beyond the Navier-Stokes equation," J. Fluid Mech. 550, 413 (2006).

${ }^{35}$ X. Nie, X. Shan, and H. Chen, "Thermal lattice Boltzmann model for gases with internal degrees of freedom," Phys. Rev. E 77, 035701(R) (2008).

${ }^{36}$ G. Goldrian, T. Huth, B. Krill, J. Lauritsen, H. Schick, I. Ouda, S. Heybrock, D. Hierl, T. Maurer, N. Meyer, A. Schafer, S. Solbrig, T. Streuer, T. Wettig, D. Pleiter, K.-H. Sulanke, F. Winter, H. Simma, S. F. Schifano, and R. Tripiccione, "Quantum chromodynamics parallel computing on the cell broadband engine," Comput. Sci. Eng. 10, 46 (2008).

${ }^{37}$ H. Baier, H. Boettiger, M. Drochner, N. Eicker, U. Fischer, Z. Fodor, A. Frommer, C. Gomez, G. Goldrian, S. Heybrock, D. Hierl, M. Hsken, T. Huth, B. Krill, J. Lauritsen, T. Lippert, T. Maurer, B. Mendl, N. Meyer, A. Nobile, I. Ouda, M. Pivanti, D. Pleiter, M. Ries, A. Schfer, H. Schick, F. Schifano, H. Simma, S. Solbrig, T. Streuer, K.-H. Sulanke, R. Tripiccione, J.-S. Vogt, T. Wettig, and F. Winter, QPACE-A QCD parallel computer based on cell processors," Proceedings of 27th International Symposium on Lattice Field Theory (2009), Pos(LAT2009) 001.

${ }^{38}$ A. Bartoloni, C. Battista, C. Cabasino, P. S. Paolucci, J. Pech, R. Sarno, G. M. Todesco, M. Torelli, W. Tross, P. Vicini, R. Benzi, N. Cabibbo, F. Massaioli, and R. Tripiccione, "LBE simulations of Rayleigh-Bènard con- vection on the APE100 parallel processor," Int. J. Mod. Phys. C 4, 993 (1993).

${ }^{39}$ E. A. Spiegel, "Convective instability in a compressible atmosphere," Astrophys. J. 141, 1068 (1965).

${ }^{40}$ E. A. Spiegel and G. Veronis, "On the Boussinesq approximation for a compressible fluid," Astrophys. J. 131, 442 (1960).

${ }^{41}$ J. R. Ristorcelli and T. T. Clark, "Rayleigh-Taylor turbulence: Self-similar analysis and direct numerical simulations," J. Fluid Mech. 507, 213 (2004).

${ }^{42}$ W. H. Cabot and A. W. Cook, "Reynolds number effects on RayleighTaylor instability with possible implications for type-Ia supernovae," Nature (London) 2, 562 (2006).

${ }^{43}$ T. T. Clark, "A numerical study of the statistics of a two dimensional Rayleigh-Taylor mixing layer," Phys. Fluids 15, 2413 (2003).

${ }^{44} \mathrm{~W}$. Cabot, "Comparison of two- and three-dimensional simulations of miscible Rayleigh-Taylor instability," Phys. Fluids 18, 045101 (2006).

${ }^{45}$ Y.-N. Young, H. Tufo, A. Dubey, and R. Rosner, "On the miscible Rayleigh-Taylor instability: Two and three dimensions," J. Fluid Mech. 447, 377 (2001).

${ }^{46}$ S. I. Abarzhi, A. Gorobets, and K. R. Sreenivasan, "Turbulent mixing in immiscible, miscible and stratified media," Phys. Fluids 17, 081705 (2005).

${ }^{47}$ S. I. Abarzhi, M. Cadjan, and S. Fedotov, "Stochastic model of the Rayleigh-Taylor turbulent mixing,” Phys. Lett. A 371, 457 (2007).

${ }^{48}$ G. Boffetta, F. De Lillo, and S. Musacchio, "Nonlinear diffusion model for Rayleigh-Taylor mixing," Phys. Rev. Lett. 104, 034505 (2010).

${ }^{49}$ J. M. Buick and C. A. Greated, "Gravity in a lattice Boltzmann model," Phys. Rev. E 61, 5307 (2000).

${ }^{50} \mathrm{Z}$. Guo, C. Zheng, and B. Shi, "Discrete lattice effects on the forcing term in the lattice Boltzmann method," Phys. Rev. E 65, 046308 (2002).

${ }^{51}$ R. Surmas, C. E. Pico Ortiz, and P. C. Philippi, "Simulating thermohydrodynamics by finite difference solutions of the Boltzmann equations," Eur. Phys. J. Spec. Top. 171, 81 (2009).

${ }^{52}$ F. Belletti, L. Biferale, F. Mantovani, S. F. Schifano, F. Toschi, and R. Tripiccione, "Multiphase lattice Boltzmann on the cell broadband engine," Nuovo Cimento Soc. Ital. Fis., C 32, 53 (2009).

${ }^{53} \mathrm{H}$. Kraichnan, "Turbulent thermal convection at arbitrary Prandtl number," Phys. Fluids 5, 1374 (1962).

${ }^{54}$ G. K. Batchelor, "Pressure fluctuations in isotropic turbulence," Proc. Cambridge Philos. Soc. 47, 359 (1951).

${ }^{55} \mathrm{C}$. Meneveau, "Transition between viscous and inertial-range scaling of turbulence structure functions," Phys. Rev. E 54, 3657 (1996).

${ }^{56} \mathrm{~S}$. Kurien and K. R. Sreenivasan, "Anisotropic scaling contributions to high-order structure functions in high-Reynolds-number turbulence," Phys. Rev. E 62, 2206 (2000).

${ }^{57}$ A. Arnèodo, R. Benzi, J. Berg, L. Biferale, E. Bodenschatz, A. Busse, E. Calzavarini, B. Castaing, M. Cencini, L. Chevillard, R. T. Fisher, R. Grauer, H. Homann, D. Lamb, A. S. Lanotte, E. Lévèque, B. Lüthi, J. Mann, N. Mordant, W.-C. Müller, S. Ott, N. T. Ouellette, J.-F. Pinton, S. B. Pope, S. G. Roux, F. Toschi, H. Xu, and P. K. Yeung (International Collaboration for Turbulence Research), "Universal intermittent properties of particle trajectories in highly turbulent flows," Phys. Rev. Lett. 100, 254504 (2008).

${ }^{58}$ R. Benzi, L. Biferale, R. Fisher, D. Q. Lamb, and F. Toschi, "Inertial range Eulerian and Lagrangian statistics from numerical simulations of isotropic turbulence,” J. Fluid Mech. 653, 221 (2010).

${ }^{59}$ J. Schumacher, "Sub-Kolmogorov-scale fluctuations in fluid turbulence," Europhys. Lett. 80, 54001 (2007).

${ }^{60} \mathrm{~V}$. Yakhot and K. R. Sreenivasan, "Towards a dynamical theory of multifractals in turbulence," Physica A 343, 147 (2004).

${ }^{61}$ R. Benzi and L. Biferale, "Fully developed turbulence and the multifractal conjecture," J. Stat. Phys. 135, 977 (2009).

${ }^{62} \mathrm{~L}$. Biferale, "A note on the fluctuation of dissipative scale in turbulence," Phys. Fluids 20, 031703 (2008).

${ }^{63}$ R. Benzi, S. Ciliberto, R. Tripiccione, C. Baudet, F. Massaioli, and S. Succi, "Extended self-similarity in turbulent flows," Phys. Rev. E 48, R29 (1993).

${ }^{64}$ R. Benzi, L. Biferale, S. Ciliberto, M. V. Struglia, and R. Tripiccione, "Generalized scaling in fully developed turbulence," Physica D 96, 162 (1996).

${ }^{65}$ L. Biferale and I. Procaccia, "Anisotropy in turbulent flows and in turbulent transport," Phys. Rep. 414, 43 (2005).

${ }^{66}$ A. Celani, A. Lanotte, A. Mazzino, and M. Vergassola, "Fronts in passive scalar turbulence," Phys. Fluids 13, 1768 (2001). 
${ }^{67}$ R. Benzi, R. Tripiccione, and F. Toschi, "On the heat transfer in RayleighBenard systems," J. Stat. Phys. 93, 901 (1998).

${ }^{68}$ E. Calzavarini, F. Toschi, and R. Tripiccione, "Evidences of BolgianoObhukhov scaling in three dimensional Rayleigh-Bénard convection," Phys. Rev. E 66, 016304 (2002).
${ }^{69} \mathrm{X}$. Shan and H. Chen, "Lattice Boltzmann model for simulating flows with multiple phases and components," Phys. Rev. E 47, 1815 (1993).

${ }^{70}$ R. Benzi, L. Biferale, M. Sbragaglia, S. Succi, and F. Toschi, "Mesoscopic modelling of a two-phase flow in presence of the boundaries: The contact angle," Phys. Rev. E 74, 021509 (2006). 